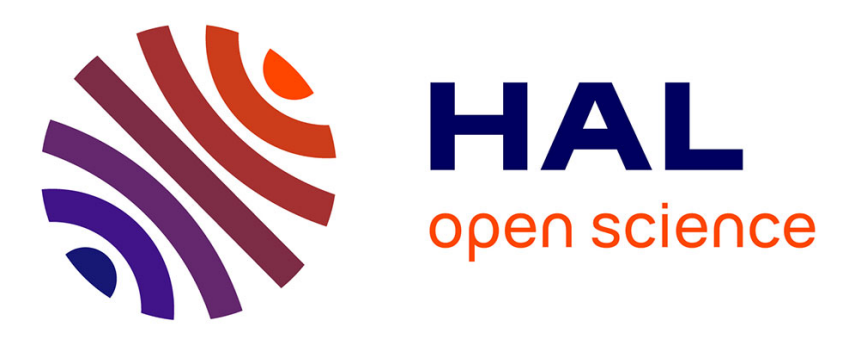

\title{
Semi-Autonomous Haptic Teleoperation Control Architecture of Multiple Unmanned Aerial Vehicles
}

Dongjun Lee, Antonio Franchi, Hyoung Il Son, Changsu Ha, Heinrich H Bülthoff, Paolo Robuffo Giordano

\section{- To cite this version:}

Dongjun Lee, Antonio Franchi, Hyoung Il Son, Changsu Ha, Heinrich H Bülthoff, et al.. Semi-Autonomous Haptic Teleoperation Control Architecture of Multiple Unmanned Aerial Vehicles. IEEE/ASME Transactions on Mechatronics, 2013, 18 (4), pp.1334-1345. 10.1109/TMECH.2013.2263963 . hal-00910801

\section{HAL Id: hal-00910801 \\ https://hal.inria.fr/hal-00910801}

Submitted on 28 Nov 2013

HAL is a multi-disciplinary open access archive for the deposit and dissemination of scientific research documents, whether they are published or not. The documents may come from teaching and research institutions in France or abroad, or from public or private research centers.
L'archive ouverte pluridisciplinaire HAL, est destinée au dépôt et à la diffusion de documents scientifiques de niveau recherche, publiés ou non, émanant des établissements d'enseignement et de recherche français ou étrangers, des laboratoires publics ou privés. 


\title{
Semi-Autonomous Haptic Teleoperation Control Architecture of Multiple Unmanned Aerial Vehicles
}

\author{
Dongjun Lee, Member, IEEE, Antonio Franchi, Member, IEEE, Hyoung Il Son, Member, IEEE, \\ ChangSu Ha, Student Member, IEEE, Heinrich H. Bülthoff, Member, IEEE, and \\ Paolo Robuffo Giordano, Member, IEEE
}

\begin{abstract}
We propose a novel semi-autonomous haptic teleoperation control architecture for multiple unmanned aerial vehicles (UAVs), consisting of three control layers: 1) UAV control layer, where each UAV is abstracted by, and is controlled to follow the trajectory of, its own kinematic Cartesian virtual point (VP); 2) VP control layer, which modulates each VP's motion according to the teleoperation commands and local artificial potentials (for VP-VP/VP-obstacle collision avoidance and VP-VP connectivity preservation); and 3) teleoperation layer, through which a single remote human user can command all (or some) of the VPs' velocity while haptically perceiving the state of all (or some) of the UAVs and obstacles. Master-passivity/slave-stability and some asymptotic performance measures are proved. Experimental results using four custom-built quadrotor-type UAVs are also presented to illustrate the theory.
\end{abstract}

Index Terms - haptic feedback, multiagent control, passivity, teleoperation, unmanned aerial vehicles

\section{INTRODUCTION}

Due to the absence of human pilots on-board, unmanned aerial vehicles (UAVs) can realize many powerful aerospace applications with reduced cost/danger and possibly higher performance than the conventional pilot-driven aerial vehicles: surveillance and reconnaissance, fire-fighting and rescue, remote sensing and exploration, pesticide spraying and geophysical survey, logistics and payload transport, and ad-hoc communication gateway, to name just few. See [1], [2]. Deploying multiple UAVs will further enhance these applications by infusing them with the benefits of multi-agent systems (e.g., better performance via cooperation such as higher payload transport and faster domain coverage; better affordability than a single/bulky system; robustness against single point failures,

Submitted to IEEE/ASME Transactions on Mechatronics, Focused Section on Aerospace Mechatronics, August 2012. Revised January 2013. Accepted April 2013

D. J. Lee and C. Ha are with the School of Mechanical \& Aerospace Engineering and IAMD, Seoul National University, Seoul, Korea, 151-744. Email: djlee@snu.ac.kr.

A. Franchi is with the Max Planck Institute for Biological Cybernetics, Spemannstraße 38, 72076, Tübingen, Germany. E-mail: antonio.franchi@tuebingen.mpg.de.

H. I. Son is with the Institute of Industrial Technology, Samsung Heavy Industries, 217 Munji-ro, Yuseong-gu, Daejeon, Korea, 305-380. Email: hi2.son@ samsung.com

H. H. Bülthoff is with the Max Planck Institute for Biological Cybernetics, Spemannstraße 38, 72076 Tübingen, Germany, and with the Department of Brain and Cognitive Engineering, Korea University, Anam-dong, Seongbukgu, Seoul, 136-713 Korea. E-mail: hhb@tuebingen.mpg.de.

P. Robuffo Giordano is with the CNRS at Irisa, Campus de Beaulieu, 35042 Rennes Cedex, France. Email: prg@irisa.fr.

Research supported in part by the Korea NRF-MEST 2009-0087640 and R31-2008-000-10008-0, and the Max Planck Society, Germany. etc.). On the other hand, many real UAV applications take place in environments, which are unstructured, uncertain and not precisely known a priori. For such cases, fully-autonomous control of the UAVs is typically infeasible/impossible, and, instead, to impose human's intelligence on the task to cope with such uncertainty, some teleoperation of their behaviors is desired, if not absolutely necessary ${ }^{1}$.

Now, suppose that a large number of UAVs is presented to a human user and $\mathrm{s} / \mathrm{he}$ is required to teleoperate their motions all at once. This would define a daunting task for the human user, since we humans can control well only a small number of degrees-of-freedom (DOFs) simultaneously, yet, such many UAVs are characterized by a large number of DOFs. However, if we examine many practical aerospace applications deploying multiple UAVs, we can also see that, very often, the task for each UAV can be split into a component, that is rather simple and mathematically well-defined (e.g., maintaining relative distance, avoid collision/obstacles, etc.) and another component, that is mathematically obscure and requires complex intelligent/cognitive information processing (e.g., how to drive UAVs in the presence of uncertainty; whether to proceed/stop when obstacles appear, etc.).

With this distinction in mind, in this paper, we propose a novel semi-autonomous haptic teleoperation control framework for multiple UAVs, which enables a single remote human user to stably teleoperate the overall (abstracted) motion of the multiple UAVs with some useful haptic feedback, while the UAVs are reacting autonomously among themselves and against local obstacles so as to render themselves collectively as a flying teleoperated deformable object. More specifically, our semi-autonomous teleoperation control architecture consists of the following three control layers (see Fig. 1):

1) UAV control layer, which enforces each UAV to (unilaterally) track the trajectory of its own first-order kinematic Cartesian virtual point (VP). For this, we assume availability of some reasonably-good trajectory tracking control law for each UAV (e.g., [3], [4], [5], [6], [7], [8], [9], or that presented in Sec. II-B), which then allow us to abstract each UAV by their kinematic VP for the purpose of their semi-autonomous teleoperation control design, while bypassing the low-level control issues of UAVs (e.g., control under-actuation [6], [7]);

\footnotetext{
${ }^{1}$ Even for highly autonomous UAVs, some form of (multisensory) feedback of the remote UAVs' state and their environment would still be beneficial for the human user (e.g., better situational awareness, telepresence).
} 
2) VP control layer, which modulates the motion of the multiple VPs in such a way that, as a whole, in a distributed manner (i.e., each VP is sensing/communicating only with their own neighboring VPs on a certain timeinvariant connectivity (or information) graph $G$ ), they collectively behave as a multi-nodal flying deformable object, whose shape autonomously deforms according to local artificial potentials (designed for VP-VP/VPobstacle collision avoidance and VP-VP connectivity maintenance), while whose bulky motion is driven by the teleoperation (velocity) command received from the master side;

3) teleoperation layer, which enables a remote human user to tele-drive some (or all) of the VPs (i.e., control set $\mathcal{N}_{c}$ ), while haptically perceiving the state of some (or all) of the real UAVs (i.e., sensing set $\mathcal{N}_{s}$ ). For this, passive set-position modulation (PSPM [10]) is adopted due to its implementation flexibility (e.g., can accommodate master-slave kinematic/dynamic dissimilarity and various forms of haptic feedback signals), guarantee of passivity (i.e., interaction stability with human users), and less conservative passifying action (thus, better performance).

Although there are numerous results for the single UAV motion control (e.g. [3]-[9]) and some results for the single UAV haptic teleoperation (e.g. [7], [11], [12], [13]), it would be fair to say that the problem of haptic teleoperation of multiple UAVs starts being considered fairly recently.

The framework of [14], which was later applied to UAVs in [15], utilizes passive decomposition [16], [17] to precisely maintain the formation shape (i.e., shape system) among the agents, while their overall motion (i.e., locked system) is teleoperated. These results [14], [15], however, demand allto-all communication among the agents and also are limited to master-slave position-position teleoperation, which is not so suitable for the UAV teleoperation, since the master workspace is bounded, yet, that of the slave UAVs is not (i.e., kinematic dissimilarity [18]). Passive decomposition, extended to nonholonomic systems [19], was also used in [20] for the teleoperation of multiple wheeled mobile robots. This work [20] has some similarity with the current work (e.g., master-position/slave-velocity teleoperation via PSPM), yet, still requires all-to-all communication and does not address collision-avoidance/connectivity-preservation among the slave robots.

To our knowledge, the works of [21], [22] are the very first results on the haptic teleoperation of multiple UAVs, that do not require all-to-all communication among the UAVs (i.e., distributed) and also consider the issues of collision and connectivity among them. Here, [21] is the conference version of the current manuscript, while [22], later expanded in [23], [24], considers the switching leader-follower information topology among the second-order dynamic VPs with constant time-delay between the master and the leader UAV. Subsequent developments/improvements ensuing these [21], [22] are as follows: 1) in [25], a partially-decentralized shared control system for multi-UAVs is proposed, that is based only on bearing (angular) measurements locally obtainable from

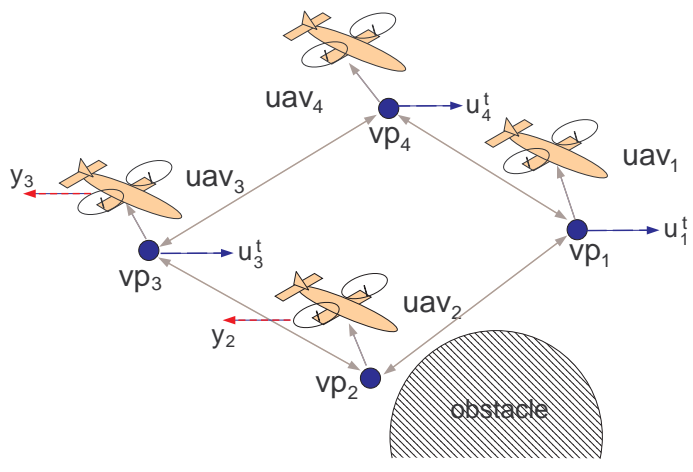

Fig. 1. Semi-autonomous haptic teleoperation with four UAVs and their VPs: gray arrows represent information flow of local autonomous UAV/VP control; blue dashed arrows velocity command for tele-control; and red doted arrows haptic feedback for tele-sensing. Here, the control set is $\mathcal{N}_{t}=\{1,3,4\}$ while the sensing set $\mathcal{N}_{s}=\{2,3\}$.

camera-like sensors; 2) in [26], a decentralized approach, that can enforce global connectivity (e.g., for steady information flow among the UAVs) in the presence of graph switching and teleoperation, is presented; and 3) in [27], by using the control framework proposed in [21] and in this paper, the impact and effectiveness of different haptic feedback for multi-UAV haptic teleoperation is studied from a perceptual point of view.

Differently from the works mentioned above [14], [15], [20], [22]-[26], our semi-autonomous haptic teleoperation control architecture, proposed first in [21] and detailed/completed in this paper, possesses the following properties: 1) the information flow (i.e., connectivity graph) among the UAVs is distributed (cf. [14], [15], [20], [25]) and their collective shape can reactively deform according to the external environment (cf. [14], [15], [20]); 2) master-passivity/slave-stability (with first-order kinematic VPs) is enforced, which is likely less conservative than master-passivity/slave-passivity of other results using the second-order dynamic VPs (cf. [14], [15], [22], [23], [24], [26]); 3) any forms of haptic feedback signal can be adopted without jeopardizing master-passivity/slavestability even in the presence of communication unreliability ${ }^{2}$ (cf. [14], [15], [22], [23], [24], [26]); and 4) the human user can freely choose any "control set" $\mathcal{N}_{t}$ and any "sensing set" $\mathcal{N}_{s}$ from multiple UAVs for tele-control/sensing depending on task objectives and conditions (cf. [14], [15], [20], [22], [23], [24], [26]). Our semi-autonomous control architecture has also served as the foundation for some of those subsequent results (e.g., kinematic VPs and flexible PSPM with masterpassivity/slave-stability: [25], [27], [7]).

A portion of this paper was presented in [21]. The current version has been substantially revised from [21], particularly with: 1) full experiment (i.e., with real UAVs) as compared to the only semi-experiment (i.e., with simulated UAVs) in [21] (Sec. III); 2) complete explanation on implementing the UAV control layer, which was only alluded in [21] (Sec. II-

\footnotetext{
${ }^{2}$ For brevity of the paper and due to the easiness of inferring how communication unreliability would affect our semi-autonomous teleoperation architecture via PSPM from [10], [28], in this paper, we omit experimental results with imperfect communication and instead refer readers to [10], [28]. The obtained theoretical results (e.g., Prop. 1, Th. 1) yet equally hold for the imperfect communication.
} 
B); and 3) whole new introduction, improved organization and significantly expanded explanations of technical results/details. Some high-level description of our semi-autonomous architecture was also reported in [29], yet, without technical details, which are fully provided in this paper.

The rest of this paper is organized as follows. Sec. II introduces some preliminary materials and details the three control layers: UAV control layer in Sec. II-B; VP control layer in Sec. II-C; and teleoperation layer in Sec. II-D. Sec. III presents experimental results using four custom-built quadrotor-type UAVs with hardware/software details. Sec. IV summarizes the paper with some comments on future research directions.

\section{Semi-Autonomous Teleoperation Control ARCHITECTURE}

\section{A. Slave UAVs and Master Haptic Device}

Let us consider $N$ UAVs, whose 3-DOF Cartesian positions are denoted by $x_{i} \in \Re^{3}, i=1,2, \ldots, N$. Here, we are interested in the case where a single human user teleoperates the Cartesian positions $x:=\left[x_{1} ; x_{2} ; \ldots ; x_{N}\right] \in \Re^{3 N}$ of the $N$ UAVs simultaneously. For this, we do not require the UAVs to be of a specific type. We rather allow them to be of any types (e.g., swarm of heterogeneous UAVs) as long as a reasonablyperforming trajectory tracking control exists for them so that we can drive each $x_{i}$ to faithfully track a smooth reference trajectory. See Sec. II-B.

One class of such UAVs, that possesses a well-performing trajectory tracking control, is so called vectored-thrust (or thrust-propelled) UAVs [6], whose 6-DOF (under-actuated) dynamics in $\mathrm{SE}(3)$ is given by

$$
\begin{aligned}
& m_{i} \ddot{x}_{i}=-\rho_{i} R_{i} e_{3}+m_{i} g e_{3}+\delta_{i} \\
& J_{i} \dot{w}_{i}+S\left(w_{i}\right) J_{i} w_{i}=\gamma_{i}+\zeta_{i}, \quad \dot{R}_{i}=R_{i} S\left(w_{i}\right)
\end{aligned}
$$

where $m_{i}>0$ is the mass, $x_{i} \in \Re^{3}$ is the Cartesian center-ofmass position w.r.t. the NED (north-east-down) inertial frame (with $e_{1}, e_{2}, e_{3}$ representing $\mathrm{N}, \mathrm{E}$ and D directions), $\rho_{i} \in \Re$ is the thrust control input along the body-frame $e_{3}, R_{i} \in S O(3)$ is the rotational matrix describing the body NED frame of UAV w.r.t. to the inertial NED frame, $w_{i} \in \Re^{3}$ is the angular rate of the UAV relatively to the inertial frame represented in the body frame, $J_{i} \in \Re^{3 \times 3}$ is the UAV's inertia matrix w.r.t. the body frame, $g$ is the gravitational constant, $\gamma_{i} \in \Re^{3}$ is the attitude torque control input, $\delta_{i}, \zeta_{i} \in \Re^{3}$ are the aerodynamic perturbations, and $S(\star): \Re^{3} \rightarrow s o(3)$ is the skew-symmetric operator defined s.t. for $a, b \in \Re^{3}, S(a) b=a \times b$. Some examples of this vectored-thrust UAV include: autonomous helicopters [4], [30], VTOL aircraft [9], ducted-fan UAVs [8] and, quadrotors [6], which we use for the experiment in Sec. III.

Let us also consider a 3-degree-of-freedom (DOF) nonlinear Lagrangian haptic device as modeled by [10], [15]

$$
M(q) \ddot{q}+C(q, \dot{q}) \dot{q}=\tau+f
$$

where $q \in \Re^{3}$ is the configuration, $M(q) \in \Re^{3 \times 3}$ is the positive-definite/symmetric inertia matrix, $C(q, \dot{q}) \in \Re^{3 \times 3}$ is the Coriolis matrix, and $\tau, f \in \Re^{3}$ are the control and human forces, respectively. It is well-known that this haptic device is (energetically) passive: $\forall T \geq 0, \exists d \in \Re$ s.t.,

$$
\int_{0}^{T}[\tau+f]^{T} \dot{q} d t=\kappa(T)-\kappa(0) \geq-\kappa(0)=: d^{2}
$$

which can be easily shown by using that $\dot{M}-2 C$ is skewsymmetric [31].

Our goal is then to enable a single remote user to teleoperate $N$ UAVs' Cartesian motions $x:=\left[x_{1} ; x_{2} ; \ldots ; x_{N}\right] \in \Re^{3 N}$ via the single 3-DOF master haptic device (3) simultaneously, while providing the user with some useful haptic feedback to convey information of the $N$ UAVs' state and their surrounding environments. There are several interesting aspects/challenges to achieve this: 1) large slave DOF: humans can usually control well only a small number of DOFs at the same time (e.g., 3-DOF master device (3)), yet, the slave $N$ UAVs possess a large number of DOFs; 2) kinematic/dynamic dissimilarity [18]: usual master device (3) has a bounded workspace with full-actuation (e.g., joystick), yet, the UAVs' workspace is unbounded (e.g., $E(3)$ ) and their dynamics (e.g., (1)-(2)) typically not so favorable to control as the fullactuated master device (e.g., under-actuated 3-DOF translation dynamics (1) with 1-DOF thrust control $\rho_{i}$ ); and 3) control distribution among UAVs: information flow (either through communication or sensing) among the UAVs is desired to be distributed (i.e., each UAV requires information only from their neighbors and possibly from the master site), particularly when the number of UAVs is large.

To address these challenges, we propose a novel semiautonomous haptic teleoperation control architecture for multiple UAVs, consisting of the three control layers, UAV control layer, VP control layer, and PSPM-based teleoperation layer, each to be detailed in the following three subsections.

\section{B. UAV Control Layer}

In this paper, we are interested in teleoperating the Cartesian motions $x=\left[x_{1} ; x_{2} ; \ldots, x_{N}\right] \in \Re^{3 N}$ of the $N$ UAVs, whose dynamics (e.g., (1)), yet, is typically too complicated to be directly handled with by the standard teleoperation techniques (e.g., under-actuation of (1)). It is also desirable in many cases to "hide" this complex underlying dynamics of the UAVs from the human user so that s/he can focus more on the high-level teleoperation of the multiple UAVs without being distracted to simultaneously taking care of their low-level dynamics.

To circumvent this issue, we endow each UAV with a $3-D O F$ Cartesian VP (virtual point), $p_{i} \in \Re^{3}$. The human user will then teleoperate these $N$ VPs instead of the real UAVs, while the real UAV (i.e., $x_{i}$ ) is tracking its own VP (i.e., $p_{i}$ ). See Fig. 1. Abstracting each UAV by their VP and formulating the semi-autonomous teleoperation objectives on these (simpler) $N$ VPs, we can greatly simplify the design of the VPcontrol layer (Sec. II-C) and of the teleoperation layer (Sec. II-D), while encapsulating the issue of the UAVs' complex low-level dynamics within the UAV control layer. This also implies that our semi-autonomous teleoperation architecture is applicable to any types of (possibly heterogeneous) mobile robots (e.g., humanoids, unicycles) as long as they possess 
some adequately-functioning tracking controller to follow their own VPs.

The vectored-thrust UAVs (1)-(2) assume many of such well-performing tracking control laws (e.g., [4], [8], [6], [7]). Although one of these schemes can certainly be used, to facilitate readers' implementation of our framework, here, we present a simple tracking control law for (1)-(2), which turns out to be fairly reliable and robust during our experiments in Sec. III and also many other demonstrations performed at the authors' institutions using quadrotor UAVs.

Our control law is based on the natural decoupling property of (1)-(2), that is, the attitude dynamics (2) is independent from the translation dynamics (1). We then design our controller to have the following inner-outer loop structure: 1) a slower outer-loop position tracking controller is designed for (1) to drive $x_{i}$ to track $p_{i}$, while specifying thrust and attitude commands; whereas 2) a faster inner-loop attitude controller is designed for (2) to attain the attitude commands given from the outer-loop. In the following derivations of the controller, for notational convenience, we omit UAV's index $i$ in (1)-(2).

First, let $\eta:=[\phi, \theta, \psi]^{T} \in \Re^{3}$ be the RPY Euler angle representation of the rotation matrix $R$, with $\phi, \theta, \psi$ being respectively the roll, pitch and yaw angles of the UAV along the NED-directions. We can then rewrite the attitude dynamics (2) using $\eta$ s.t.

$$
\Sigma_{1}:\left\{\begin{aligned}
J \dot{w} & =-S(w) J w+\gamma+\zeta \\
\dot{\eta} & =T(\eta) w
\end{aligned}\right.
$$

where $T(\eta) \in \Re^{3 \times 3}$ is the transformation matrix from $w \in$ so(3) to the Euler angle rates $\dot{\eta}$. The translational dynamics (1) can also be written by using $\eta$ s.t.

$$
\Sigma_{2}: m \ddot{x}=m g e_{3}-\rho R(\eta) e_{3}+\delta
$$

where $R(\eta)=R_{e_{3}}(\psi) R_{e_{2}}(\theta) R_{e_{1}}(\phi)$, with $R_{e_{i}}$ being the elementary rotation matrix about the $e_{i}$-axis [31].

Notice that the attitude dynamics $\Sigma_{1}$ is independent from $\Sigma_{2}$ (and $\rho$, which will contain the control action for $x$ ), while the reverse does not hold, i.e., $\Sigma_{2}$ depends on $\Sigma_{1}$ due to the term $R(\eta)$. The goal of the controller is then to make use of the four control inputs, $\gamma \in \Re^{3}$ and $\rho \in \Re$, to separately control the UAV's position $x=\left(x_{1}, x_{2}, x_{3}\right)^{T}$ to track a (smooth) VP's reference trajectory $p=\left(p_{1}, p_{2}, p_{3}\right)^{T}$ and the yaw angle $\psi$ to (possibly time-varying) target value $\psi_{d}$.

Let us start with the position controller first. For this, using $R(\eta)=R_{e_{3}}(\psi) R_{e_{2}}(\theta) R_{e_{1}}(\phi)$, we can write (5) as

$$
R_{e_{3}}^{T}(\psi) m \ddot{x}=m g e_{3}-R_{e_{2}}(\theta) R_{e_{1}}(\phi) \rho e_{3}+R_{e_{3}}^{T}(\psi) \delta
$$

whose last row reads s.t.

$$
m \ddot{x}_{3}=m g-\cos (\phi) \cos (\theta) \rho+\delta_{3}
$$

with $\delta=\left(\delta_{1}, \delta_{2}, \delta_{3}\right)^{T}$. This then suggests the following thrust control

$$
\rho=-\frac{m}{\cos \phi \cos \theta}\left[-g+\ddot{p}_{3}+k_{d_{P}}\left(\dot{p}_{3}-\dot{x}_{3}\right)+k_{p_{P}}\left(p_{3}-x_{3}\right)\right]
$$

which ensures local exponential stability of $p_{3}-x_{3}$, as long as the system is away from the singularity $\cos \phi \cos \theta=0$ and $\delta_{3}=0$.
On the other hand, the first two rows of (5) are given by

$$
m\left(\begin{array}{c}
\ddot{x}_{1} \\
\ddot{x}_{2}
\end{array}\right)=-\rho \underbrace{\left[\begin{array}{cc}
\cos \phi \cos \psi & \sin \psi \\
\cos \phi \sin \psi & -\cos \psi
\end{array}\right]}_{=: Q(\phi, \psi) \in \Re^{2 \times 2}}\left(\begin{array}{c}
\sin \theta \\
\sin \phi
\end{array}\right)+\left(\begin{array}{c}
\delta_{1} \\
\delta_{2}
\end{array}\right)
$$

where $Q$ is always analytically invertible as long as $\cos \phi \neq 0$. This then shows that $\left(p_{1}-x_{1}, p_{2}-x_{2}\right)$ will be locally exponentially stable (with $\delta_{1}=\delta_{2}=0$ ), if the attitude controller (to be defined below) can attain pitch and roll commands $\theta_{d}, \phi_{d}$ given by

$$
\left(\begin{array}{c}
\sin \theta_{d} \\
\sin \phi_{d}
\end{array}\right)=\frac{m Q^{-1}}{-\rho}\left(\begin{array}{l}
\ddot{p}_{1}+k_{d_{P}}\left(\dot{p}_{1}-\dot{x}_{1}\right)+k_{p_{P}}\left(p_{1}-x_{1}\right) \\
\ddot{p}_{2}+k_{d_{P}}\left(\dot{p}_{2}-\dot{x}_{2}\right)+k_{p_{P}}\left(p_{2}-x_{2}\right)
\end{array}\right) .
$$

Here, although (8) defines a nonlinear equation for $\phi$, we found it works pretty well in practice to obtain $\left(\theta_{d}, \phi_{d}\right)$ while assuming $Q$ be a function of $(\phi, \psi)$, particularly with fast enough attitude control servo-rate (e.g., $500 \mathrm{~Hz}$ in Sec. III).

Now, define the desired attitude set-point by $\eta_{d}:=$ $\left[\phi_{d}, \theta_{d}, \psi_{d}\right]$, where $\phi_{d}, \theta_{d}$ are given above from the (outerloop) position controller (8), while $\psi_{d}$ can be set arbitrarily (e.g., $\psi_{d}=0$ ). Given $\eta_{d}$, we then design the (fast/inner-loop) attitude regulation control as follows. First, differentiating the second row of (4), we can get

$$
\ddot{\eta}=T(\eta) \dot{w}+\dot{T}(\eta) w
$$

which, by using the first row of (4), becomes

$$
\ddot{\eta}=T(\eta) J^{-1}(-w \times J w+\gamma+\zeta)+\dot{T}(\eta) w .
$$

We then choose the attitude regulation control s.t.

$$
\gamma=J T^{-1}(\eta)\left(-k_{d_{A}} \dot{\eta}+k_{p_{A}}\left(\eta_{d}-\eta\right)\right)
$$

with which we have the following closed-loop dynamics:

$$
\ddot{e}_{\eta}+\left[k_{d_{A}}+T J^{-1} S(w) J w-\dot{T} T^{-1}\right] \dot{e}_{\eta}+k_{p_{A}} e_{\eta}=T J^{-1} \zeta
$$

with $e_{\eta}:=\eta-\eta_{d}$, implying that, if $\zeta=0,\left(e_{\eta}, \dot{e}_{\eta}\right)$ will be locally exponentially stable, if $\left(e_{\eta}(0), \dot{e}_{\eta}(0)\right)$ is small enough and $k_{d_{A}}$ large enough.

The presented controller (7)-(9), although simple in its structure (i.e., easier to implement than [4], [8], [6], [7]), turns out to be sufficient for our experiments (and many on-site demonstrations) as evidenced in Sec. III. Thus, from now on, we assume that: 1) we have implemented for each UAV a trajectory tracking control to make $x_{i}$ to follow $p_{i}$; and 2) this control performs reasonably well, by keeping $\left\|x_{i}-p_{i}\right\|$ and $\left\|\dot{x}_{i}-\dot{p}_{i}\right\|$ small enough $\left(\|x\|^{2}:=x^{T} x\right)$. How to control the motion of $N$ VPs is then the subject of the next Sec. II-C.

\section{Distributed VP Control Layer}

We consider $N$ first-order kinematic VPs to abstract each UAV, with their Cartesian position denoted by $p_{i} \in \Re^{3}$ $(i=1,2, \ldots, N)$. Our goal is to render these $N$ VPs as a $N$-nodes flying deformable object in a distributed manner, so that their shape autonomously deforms reacting to the presence of obstacles, while their collective motion is tele-controlled by a single remote human user, with the information-flow among the VPs distributed. See Fig. 1. As long as the UAV control 
layer in Sec. II-B ensures $\left\|x_{i}-p_{i}\right\|$ and $\left\|\dot{x}_{i}-\dot{p}_{i}\right\|$ be small, these VPs' behaviors will be then faithfully duplicated among the real UAVs.

To describe how the VPs are connected (via communication or sensing) to form the $N$-nodes flying object, we define the undirected connectivity graph $G$, with the $N$ VPs as its nodes and their connection $(i, j)$ as its edges. Since $G$ is undirected, $(i, j) \in \mathcal{E}(G)$ iff $(j, i) \in \mathcal{E}(G)$, where $\mathcal{E}(G)$ is the edge set of $G$. We assume $G$ is connected and also dense enough so that, with some suitably-defined inter-VP attractive/repulsive potentials on $\mathcal{E}(G)$, it can define the un-deformed shape of the $N$-nodes deformable object with no inter-VP separation or collision (e.g., rigid graph [32], [33]). We also assume $G$ to be time-invariant (e.g., no creation/elimination of edges over time). For some applications, a time-varying $G$ may be useful (e.g., separation of $N$-nodes flying object to penetrate narrow passages and merge afterwards). See [22], [23], [24], [26], where such a time-varying $G$ is achieved for second-order dynamic VPs under leader-follower connectivity graph.

We implement the following kinematic evolution of VP on each UAV: for the $i^{\text {th }} \mathrm{UAV}$,

$$
\dot{p}_{i}(t):=u_{i}^{t}+u_{i}^{c}+u_{i}^{o}
$$

where

1) $u_{i}^{c} \in \Re^{3}$ embeds the inter-VP collision avoidance and connectivity preservation, as defined by

$$
u_{i}^{c}:=-\sum_{j \in \mathcal{N}_{i}} \frac{\partial \varphi_{i j}^{c}\left(\left\|p_{i}-p_{j}\right\|^{2}\right)^{T}}{\partial p_{i}}
$$

where $\varphi_{i j}^{c}$ is a certain artificial potential function to create attractive action if $\left\|p_{i}-p_{j}\right\|$ is large, and repulsive action if $\left\|p_{i}-p_{j}\right\|$ small (see Fig. 2), and

$$
\mathcal{N}_{i}:=\{j \mid(j, i) \in \mathcal{E}(G)\}
$$

i.e., the connectivity neighbors of the $i^{\text {th }}$ VP on $G$;

2) $u_{i}^{o} \in \Re^{3}$ is the obstacle avoidance action as given by

$$
u_{i}^{o}:=-\sum_{r \in \mathcal{O}_{i}} \frac{\partial \varphi_{i r}^{o}\left(\left\|p_{i}-p_{r}^{o}\right\|\right)^{T}}{\partial p_{i}}
$$

where $\mathcal{O}_{i}$ is the set of obstacles of the $i$-th VP with $p_{r}^{o}$ being the position of the $r^{\text {th }}$ obstacle in $\mathcal{O}_{i}$, and $\varphi_{i r}^{o}$ is a certain artificial potential, which produces repulsive action if $\left\|p_{i}-p_{r}^{o}\right\|$ is small, smoothly converges to zero as $\left\|p_{i}-p_{r}^{o}\right\| \rightarrow d$, and stays zero for $\left\|p_{i}-p_{r}^{o}\right\| \geq d$, to make the effect of obstacles for each VP gradually emerge/disappear when they move closer/farther from the VP than $d>0$ (see Fig. 2);

3) $u_{i}^{t} \in \Re^{3}$ will contain the teleoperation command for the UAVs in the "control set" $\mathcal{N}_{t} \subset\{1,2, \ldots, N\}$, to enable a remote human user to directly tele-drive the Cartesian velocity of these VPs in $\mathcal{N}_{t}$ and, consequently, the collective velocity of the $N$-nodes flying deformable object (to be designed in Sec. II-D).

Each UAV numerically integrates this kinematic evolution equation (10) over the sampling time (e.g., $150 \mathrm{~Hz}$ for Sec. III) to obtain (and track) the position $p_{i}$ of their own VP. Here, both the inter-VP potential $\varphi_{i j}^{c}$ and the VP-obstacle

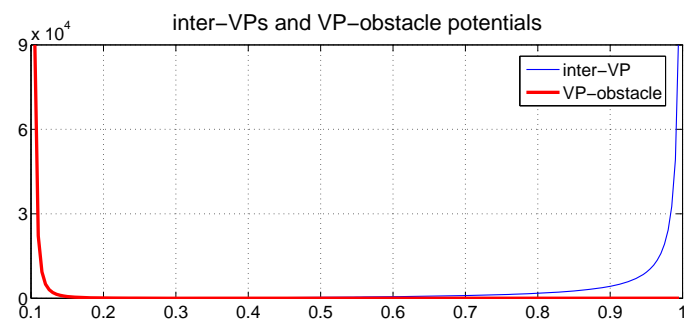

Fig. 2. Examples of $\varphi_{i j}^{c}$ and $\varphi_{i r}^{o}$, to ensure VP-VP/VP-obstacle collision distance $>0.1$ and VP-VP separation distance $<1$ [15], [34].

potential $\varphi_{i j}^{o}$ are designed s.t.: 1) they are distance-based (i.e. $\left.\varphi_{i j}^{c}\left(|| p_{i}-p_{j} \|\right)\right)$, not vector-based (i.e. $\left.\varphi_{i j}^{c}\left(p_{i}-p_{j}\right)\right)$, to allow for the rotational symmetry [35] of the $N$-VPs deformable flying object (see Sec. III-D); and 2) they rapidly increase when inter-VP/VP-obstacle collisions or inter-VP separation (e.g., with limited communication range) are impending to prevent that. For a design example of $\varphi_{i j}^{c}, \varphi_{i r}^{o}$, see [15], [34].

The next Prop. 1 summarizes some key properties of the swarm behavior of the $N$ VPs (10), with $\varphi_{i j}^{c}, \varphi_{i r}^{o}$ and bounded $u_{i}^{t}$. For that, define the total potential energy s.t.

$V(t):=\frac{1}{2} \sum_{i=1}^{N} \sum_{j \in \mathcal{N}_{i}} \varphi_{i j}^{c}\left(\left\|p_{i}-p_{j}\right\|\right)+\sum_{i=1}^{N} \sum_{r \in \mathcal{O}_{i}} \varphi_{i r}^{o}\left(\left\|p_{i}-p_{r}^{o}\right\|\right)$

and also assume that $\varphi_{i j}^{c}$ and $\varphi_{i r}^{o}$ are constructed s.t.: 1) there exists a large enough $\bar{M}>0$ s.t. $V(t) \leq \bar{M}$ implies interVP connectivity preservation and no inter-VP/VP-obstacle collisions (e.g., rigid graph [32], [33]); and 2) $\partial \varphi_{i j}^{c} / \partial p_{i}$ and $\partial \varphi_{i r}^{o} / \partial p_{i}$ are bounded, if $\varphi_{i j}^{c}$ and $\varphi_{i r}^{o}$ are bounded.

Proposition 1 Suppose $u_{i}^{t}$ is bounded with $\left\|u_{i}^{t}\right\| \leq \bar{u} \forall t \geq 0$, $\forall i \in \mathcal{N}_{t} \subset\{1,2, . ., N\}$ and $V(0)<\bar{M}$. Suppose further that, if $V(t) \geq \bar{M}$, there exists at least one $V P$, say the $s^{\text {th }} V P$, $s \in\{1,2, \ldots, N\}$, s.t.

$$
\left\|\sum_{j \in \mathcal{N}_{s}} \frac{\partial \varphi_{s j}^{c}}{\partial p_{s}}+\sum_{r \in \mathcal{O}_{s}} \frac{\partial \varphi_{s r}^{o}}{\partial p_{s}}\right\| \geq \frac{\sqrt{N_{t}}+\delta_{s t}}{2} \bar{u}
$$

where $N_{t}$ is the cardinality of $\mathcal{N}_{t}$; and $\delta_{s t}=1$ if $s \in \mathcal{N}_{t}$, and $\delta_{s t}=0$ otherwise. Then, all the NVPs are stable with bounded $\dot{p}_{i} ; V P$-VP/VP-obstacle collisions are avoided; and $V P$-VP connectivity is preserved. Moreover, if $u_{i}^{o} \equiv 0 \forall i=$ $1,2, \ldots, N$ (i.e., no obstacles) and $u_{i}^{t}$ is the same for all the UAVs in $\mathcal{N}_{t}$, we have $\sum_{i=1}^{N} \dot{p}_{i}=N_{t} \cdot u_{i}^{t}, \forall t \geq 0$.

Proof: Here, we only provide a sketch of proof. Refer to [21] for some more details. Differentiating the above $V(t)$ with $\sum_{i=1}^{N} \sum_{j \in \mathcal{N}_{i}} \frac{\partial \varphi_{i j}^{c}}{\partial p_{i}} \dot{p}_{i}=\sum_{i=1}^{N} \sum_{j \in \mathcal{N}_{i}} \frac{\partial \varphi_{i j}^{c}}{\partial p_{j}} \dot{p}_{j}$, we have

$$
\dot{V}=\sum_{i=1}^{N} W_{i}^{T}\left(-W_{i}+u_{i}^{t}\right) \leq \sum_{i=1}^{N}\left(-\left\|W_{i}\right\|^{2}+\delta_{i t} \bar{u}\left\|W_{i}\right\|\right)
$$

where $W_{i}:=-u_{i}^{c}-u_{i}^{o}$. Now, suppose that $V(t) \geq \bar{M}$. Then, from (13), $\exists s \in\{1,2, \ldots, N\}$ s.t. $\left\|W_{s}\right\| \geq \bar{u}\left(\sqrt{N_{t}}+\right.$ $\left.\delta_{s t}\right) / 2$. We can thus obtain $\dot{V} \leq-\sum_{i \notin \mathcal{N}_{t} \cup\{s\}}\left\|W_{i}\right\|^{2}-$ $\left\|W_{s}\right\|^{2}+\delta_{s t} \bar{u}\left\|W_{s}\right\|-\sum_{i \in \mathcal{N}_{t} \backslash\{s\}}\left(\left\|W_{i}\right\|^{2}-\bar{u}\left\|W_{i}\right\|\right) \leq$ $-\left(\left\|W_{s}\right\|-\frac{\delta_{s t} \bar{u}}{2}\right)^{2}+N_{t} \frac{\bar{u}^{2}}{4} \leq 0$, where we use the facts 
that $\delta_{s t}^{2}=\delta_{s t},-\left\|W_{i}\right\|^{2}+\bar{u}\left\|W_{i}\right\| \leq \bar{u}^{2} / 4$, and $\delta_{s t}^{2} \bar{u}^{2}+$ $\sum_{i \in \mathcal{N}_{t} \backslash\{s\}} \bar{u}^{2}=N_{t} \bar{u}^{2}$. This then implies $V(t) \leq \bar{M} \forall t \geq 0$, proving no inter-VP/VP-obstacle collision and inter-VP separation. Boundedness of $\dot{p}_{i}$ follows from (10), with bounded $u_{i}^{c}, u_{i}^{o}$ and $u_{i}^{t}$. The last assertion of Prop. 1 can be shown by summing up (10) for all VPs with $u_{i}^{o} \equiv 0$.

The assumption (13) of Prop. 1 is mild, since it just rules out the practically improbable (e.g., zero-measure) situation, where, although $V(t)$ is very large, none of the VPs can detect that, with all of their (also very large) forces, $\partial \varphi_{i j}^{c} / \partial p_{i}$ and $\partial \varphi_{i r}^{o} / \partial p_{i}$, somehow exactly aligned with each other to make their sum nonetheless to be small. Prop. 1 also says that: 1) all the $N$ VPs is guaranteed to be stable (i.e., bounded $\dot{p}_{i}$ and no collisions/separations) for any bounded $u_{i}^{t}$ regardless of whether $u_{i}^{t}$ are heterogeneous among the VPs or applied to some or all of them; and 2) the more VPs implement $u_{i}^{t}$, the easier it would be to drive the $N$-VPs deformable flying object. In the next Sec. II-D, this Prop. 1 will allow us to enforce master-passivity/slave-stability of the total closed-loop teleoperation system, with passive set-position modulation (PSPM [10]) robustly guaranteeing the boundedness of $u_{i}^{t}$ and master-side passivity. See [36], where a result similar to Prop. 1 was achieved by using a sliding mode control approach.

Our usage of VPs is inspired by [12]. Yet, instead of the second-order dynamic VPs in [12], [22], [23], [24], [26], here, we choose the simpler first-order kinematic VPs (10), since 1) we can significantly simplify/strengthen the VPs' swarm control design/analysis (e.g., stability of Prop. 1 valid for any bounded $u_{i}^{t}$ applied to any VPs); and 2) we can avoid some performance-limiting aspects encountered with the dynamic VPs (e.g., operator's continuous exercising against system/control damping [12]). As shown in Sec. II-D, this kinematic VP will also allow us to achieve master-passivity/slave-stability, which is less conservative than master-passivity/slave-passivity typical for the dynamic VPs [12], [22], [23], [24], [26], thus, would likely provide a sharper performance than achievable with the dynamic VPs. See [37], [38] for other "kinematic" abstractions. Our usage of VPs (10) also shares a similarity with the multi-nodal deformable object modeling or distributed behavioral swarm modeling in computer graphics (e.g., [39], [40]), in which, yet, (useful/important) theoretical guarantees as obtained here (e.g, stability with no collision/separation of Prop. 1; masterpassivity/slave-stability of Th. 1) are typically missing.

\section{PSPM-Based Teleoperation Layer}

For the teleoperation layer, we utilize passive set-position modulation (PSPM [10]), whose passifying action theoretically guarantees master-passivity/slave-stability of the closed-loop teleoperation system, while whose flexibility allows us to accommodate kinematic/dynamic dissimilarity between the master device (3) and the VPs (10) and also various forms of haptic feedback. PSPM also exhibits better performance than other 'time-invariant' teleoperation schemes (e.g. wave/PD) due to its less conservative "selective" passifying action. Our treatment on PSPM here is brief: see [10], [28] for more details.
First, to enable a remote human user to teleoperate the VPs in the control set $\mathcal{N}_{t}$, we define $u_{i}^{t}(t)$ in (10) s.t.,

$$
u_{i}^{t}(t):=\lambda H[q(k)], \quad \forall i \in \mathcal{N}_{t}
$$

for $t \in\left[t_{k}, t_{k+1}\right)$, where $q(k)$ is the master configuration $q(t) \in \Re^{3}$ received from the communication (e.g., Internet) by the $i^{\text {th }} \mathrm{UAV}$ at the reception time $t_{k}, \lambda>0$ is to match different scales between $q$ and $\dot{p}_{i}$, and $H[\cdot]$ is a continuoustime (BIBO) stable low-pass filter with a fast enough timeconstant to ensure $u_{i}^{t}(t)$ be smooth (to obtain $\ddot{p}$ for, e.g., (7)-(8)) while tracking $\lambda q(k)$ quickly enough. By providing coupling between the VPs' velocity $\dot{p}_{i}$ and master's position $q$, this control (14) allows us to address the issue of master-slave kinematic dissimilarity [18] (i.e., mobile VPs with unbounded workspace; master device with bounded workspace).

On the other hand, to allow the remote user to tele-sense some (or all) of the UAVs and their surrounding obstacles, we design the haptic feedback $y(t) \in \Re^{3}$ s.t.

$$
y(t):=\frac{1}{\lambda N_{s}} \sum_{i \in \mathcal{N}_{s}} \underbrace{\left(\dot{x}_{i}+u_{i}^{o}\right)}_{=: y_{i}(t)}
$$

where $\mathcal{N}_{s} \subset\{1,2, \ldots, N\}$ is the "sensing" set among the $N$ UAVs with $N_{s}>0$ being its cardinality, $\dot{x}_{i}$ is the $i^{\text {th }}$ UAV's velocity (1), and $u_{i}^{o}$ is the $i^{\text {th }}$ VP's obstacle avoidance control (12). This $y(t)$ is designed to allow the user: 1) to directly perceive the state of the real UAVs, thereby, completes the "information closed-loop" (i.e. master $\rightarrow$ VPs $\rightarrow$ UAVs $\rightarrow$ master) to overcome the unilaterality of the UAV control layer (e.g., prevent the user from tele-driving the VPs without knowing the UAVs are left behind due to, e.g., actuator failures) and also; 2) to tele-sense the presence of obstacles through their collective effects (i.e., $\left(1 /\left(\lambda N_{s}\right)\right) \sum_{i \in \mathcal{N}_{s}} u_{i}^{o}$ ) on the VPs/UAVs in the sensing set $\mathcal{N}_{s}$.

Each UAV in the sensing set $\mathcal{N}_{s}$ then sends its $y_{i}(t)$ (15) to the master site over communication network (e.g., Internet). Let us denote by $y(k):=\left(1 /\left(\lambda N_{s}\right)\right) \sum_{i \in \mathcal{N}_{s}} y_{i}(k)$ the haptic signal $y(t)$ constructed in the master side at the reception time $t_{k}$. We incorporate this $y(k)$ into the teleoperation control $\tau$ in (3) s.t.: for $t \in\left[t_{k}, t_{k+1}\right.$ ),

$$
\tau(t):=-B \dot{q}-K_{f} q-K(q-\bar{y}(k))
$$

where $B, K, K_{f} \in \Re^{3 \times 3}$ are the positive-(semi)definite diagonal gain matrices, and $\bar{y}(k)$ is the PSPM-modulation of the received haptic feedback $y(k)$ (to be defined below). This haptic control (16) is designed s.t.: 1) if the UAVs fleet reaches the commanded velocity, the user will perceive this steadystate UAVs' velocity haptically (via $f$ in (3)) and/or visually (by seeing q); and 2) if the UAVs fleet approaches obstacles, the user will haptically perceive these obstacles through their collective action $\sum_{i=1}^{N} u_{i}^{o}$ through the haptic feedback $y$. See item 2 of Th. 1.

At each $t_{k}$, PSPM-modulation $\bar{y}(k)$ in (16) is defined by

$$
\begin{aligned}
& \min _{\bar{y}(k)}\|y(k)-\bar{y}(k)\| \\
& \text { subj. } E(k)=E(k-1)+D_{\min }(k-1)-\Delta \bar{P}(k) \geq 0
\end{aligned}
$$

that is, $\bar{y}(k)$ is chosen as close to $y(k)$ as possible for performance (first line), yet, only to the extent permissible by 
the passivity constraint (second line). Here, $E(k) \geq 0$ is the virtual energy reservoir (simulated in software); $D_{\min }(k):=$ $\frac{1}{t_{k+1}-t_{k}} \sum_{i=1}^{3} b_{i}\left(\bar{q}_{i}(k)-\underline{q}_{i}(k)\right)^{2}$ is (conservative) estimate of the (otherwise-wasted) energy dissipation via the damping $B$ in (16), which is re-harvested into $E(k)$, with $b_{i}>0$ being the $i^{\text {th }}$ diagonal element of $B(i=1,2,3), q_{i}$ the $i^{\text {th }}$ element of $q$, and $\bar{q}_{i}(k)$ and $\underline{q}_{i}(k)$ respectively the maximum and minimum of $q_{i}(t)$ during $\left[t_{k}, t_{k+1}\right)$; and, with $\|x\|_{A}^{2}:=x^{T} A x$, $\Delta \bar{P}(k):=\left\|q\left(t_{k}\right)-\bar{y}(k)\right\|_{K}^{2} / 2-\left\|q\left(t_{k}\right)-\bar{y}(k-1)\right\|_{K}^{2} / 2$ is the energy jump at $t_{k}$, which is passified by choosing $\bar{y}(k)$ to satisfy the passivity constraint (second line).

This PSPM is implemented only on the master side. Since the human operator usually keeps injecting energy into the master system, $E(k)$ may keep increasing as well. To avoid this excessive energy accumulation in $E(k)$, we ceil off $E(k)$, by discarding any energy over a certain upper limit $\bar{E}$. Note that, if we utilize $y(k)$ directly in (16), passivity would be in general violated, since the switchings of the (discrete) signal $y(k)$ can induce (possibly-destabilizing) energy jumps in the system [10] and also the frequency/phase contents of $y(k)$ itself may not define a passive mapping with $\dot{q}$ via $K, K_{f}$. See [10], [28] for more details on PSPM. We now present the main result of this paper, whose proof we omit here and refer readers to a similar proof in [21], that can be easily applied to this Th. 1 while recognizing the BIBO stability of $H(s)$ in (14) and non-zero $K_{f}$ in (16).

Theorem 1 Consider N VPs (10) and master device (3) with PSPM-modulated teleoperation control (16).

1) Closed-loop master system is passive, i.e., $\exists d_{1} \in \Re$ s.t.,

$$
\int_{0}^{T} f^{T} \dot{q} d t \geq-d_{1}^{2}
$$

$\forall T \geq 0$. Moreover, if the assumptions of Prop. 1 hold and human user is passive, i.e., $\exists c_{2} \in \Re$ s.t.,

$$
\int_{0}^{T} f^{T} \dot{q} d t \leq c_{2}^{2}
$$

$\forall T \geq 0$, all the VPs are stable (with bounded $\dot{p}_{i}$ ) with no VP-VP/VP-obstacle collision and VP-VP separations, and $(\dot{q}, q, q-\bar{y}(k))$ are also all bounded $\forall t \geq 0$.

2) Suppose further that $(\ddot{q}, \dot{q}) \rightarrow 0, E(k)>0 \forall k \geq 0$, $\left(x_{i}(t), \dot{x}_{i}(t)\right) \rightarrow\left(p_{i}(t), \dot{p}_{i}(t)\right)$ and $\mathcal{N}_{s}=\{1,2, \ldots, N\}$. Then, (a) if $u_{i}^{o}=0, i=1,2, \ldots, N$ (i.e., no obstacles), the human user will have collective haptic velocity perception with

$$
\begin{aligned}
q(t) & \rightarrow \frac{1}{\lambda N_{t}} \sum_{i=1}^{N} \dot{x}_{i} \\
f(t) & \rightarrow\left[\frac{K_{f}}{\lambda N_{t}}+\frac{K}{\lambda} \frac{N_{s}-N_{t}}{N_{t} N_{s}}\right] \sum_{i=1}^{N} \dot{x}_{i}
\end{aligned}
$$

and (b) if $\dot{x}_{i}=0, i=1,2, \ldots, N$ (e.g., stopped by obstacles), human will have collective obstacle haptic perception with

$$
\begin{aligned}
q(t) & \rightarrow-\frac{1}{\lambda N_{t}} \sum_{i=1}^{N} u_{i}^{o} \\
f(t) & \rightarrow-\left[\frac{K_{f}}{\lambda N_{t}}+\frac{K}{\lambda} \frac{N_{s}+N_{t}}{N_{t} N_{s}}\right] \sum_{i=1}^{N} u_{i}^{o} .
\end{aligned}
$$

The results of Th. 1 still hold even when the masterslave (discrete) communication is imperfect (e.g., Internet with varying-delay, packet-loss, data-swapping, etc), since: 1) the PSPM can passify any discrete data sequence $y(k)$ regardless of how imperfect its transmission is; and 2) Prop. 1 requires only the boundedness of $u_{i}^{t}(t)$, which is guaranteed by the PSPM's ensuring boundedness of $q(t)$ robustly at the master site (with passive human assumption (18) and stability of $H$ (14)).

Due to the same reason, with PSPM, we can utilize any arbitrary forms of haptic feedback $y(t)$ other than (15) while preserving master-passivity/slave-stability (e.g., $y:=\dot{x}_{l}+$ $1 /(\lambda N) \sum_{i=1}^{N} u_{i}^{o}$, where $l$ represents a leader agent; or even nonlinear function $\left.y:=y\left(\dot{x}_{i}, u_{i}^{o}\right)\right)$. Such a flexibility is unusual with typical passivity-enforcing schemes (e.g. wave/PD) and has been exploited in [25], [27], [7]. We also performed, in [27], a psychophysical study on three different haptic feedback forms for (15) (i.e., $y=\left(1 /\left(\lambda N_{s}\right)\right) \sum_{i \in \mathcal{N}_{s}} \dot{x}_{i}$, $y=\left(1 /\left(\lambda N_{s}\right)\right) \sum_{i \in \mathcal{N}_{s}} u_{i}^{o}$, and (15)) using novel humanperspective performance measures (i.e., maneuverability and perceptual sensitivity), and revealed their respective effectiveness for specific task objectives. See [27] for more details on this psychophysical evaluation on different haptic feedback for (15). See also [41] for a psychophysical experiment on the contribution of haptic feedback itself to the user's performance improvement.

The item 2 of Th. 1 illustrates that, on top of haptic feedback, the human user will also have visual feedback, which turns out to be quite useful in some situations (i.e., seeing the device-tip position $q$, proportional to $\sum_{i=1}^{N} \dot{x}_{i}$ (19) or $\left.\sum_{i=1}^{N} u_{i}^{o}(21)\right)$. For instance, if we choose $\mathcal{N}_{s}=\mathcal{N}_{t}$ and $K_{f}=0$ as done in Sec. III-D, although $f(t) \rightarrow 0$ with $u_{i}^{o}=0$ from (20) (i.e., no force feedback in steady-state), with the visual feedback of $q(t)$ providing the velocity command information and the transient (non-zero) force feedback of $f(t)$ conveying the information of the mismatch between the velocity command and $\sum_{i=1}^{N} \dot{x}_{i}$, the human user can still adequately perceive the collective behavior of multiple UAVs and utilize it for teleoperating them. See Sec. III-D. Of course, if desired, by setting $K_{f} \neq 0$, we can easily recover non-zero steady-state force feedback even with $\mathcal{N}_{t}=\mathcal{N}_{s}$ (see (20)).

Due to the limited master device DOF, there is an unavoidable ambiguity in our haptic feedback, that is, the same pair of $(q(t), f(t))$ may correspond to the velocity information $\sum_{i=1}^{N} \dot{x}_{i}$ or to the obstacle information $\sum_{i=1}^{N} u_{i}^{o}$. This ambiguity can be addressed: 1 ) by providing a visual cue to convey the relative importance between $\sum_{i=1}^{N} \dot{x}_{i}$ and $\sum_{i=1}^{N} u_{i}^{o}$; or 2) by scaling the haptic feedback of $\sum_{i=1}^{\bar{N}_{1}} u_{i}^{o}$ much larger/steeper than that of $\sum_{i=1}^{N} \dot{x}_{i}$ so that, whenever the obstacle action is present, its (steady-state) effect can be dominant. This latter 

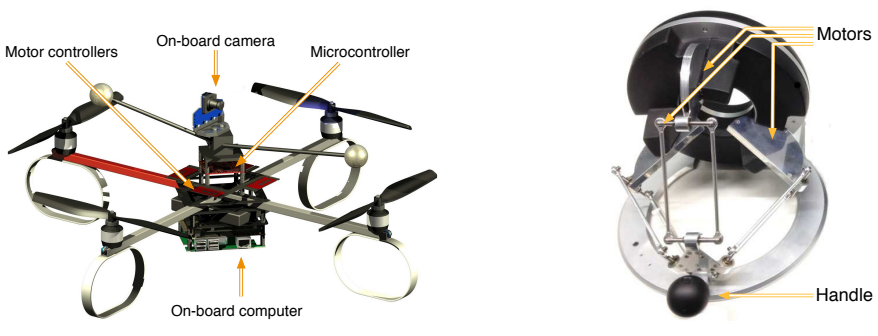

Fig. 3. On the left: our quadrotor UAV setup with its avionics parts. On the right: haptic interface used in the experimental testbed.

option is adopted for our experiment in Sec. III-D.

We also believe that the master-passivity/slave-stability (with kinematic VPs (10)) of item 1 of Th. 1 is more suitable for our purpose than the usual master-passivity/slavepassivity, since 1) it does not require the human user to, e.g., continuously overcome damping dissipation in the (dynamic) VPs simulation and UAVs dynamics (e.g., wind drag [12]); and 2) enforcing slave-passivity is not so important here (since VPs are not physically interacting with unknown environment) and rather likely detrimental (i.e., unnecessarily enforce conservative passivity both for the master and slave sides).

\section{EXPERIMENTAL TEST-Bed AND REsults}

\section{A. Hardware Setup}

The experiments reported in this section were run on a customized version of the $M K$-Quadro ${ }^{3}$, an open-source platform (see Fig. 3). Four propellers of diameter 0.254[m] are attached to four Roxxy 2827-35 motors, each of which driven via a PWM signal by a BL-Ctrl V2.0 brushless controller. The average power consumption sustainable by the controller is $160[\mathrm{~W}]$ and the peak current is $40[\mathrm{~A}]$. The motors are mounted at the end of 4 aluminum rods joined together in a cross-shape by 2 plastic center-plates. The total span and weight of the frame are $0.5[\mathrm{~m}]$ and $0.12[\mathrm{~kg}]$, respectively.

We identified the static and dynamical characteristics of the motor/propeller system by means of Nano17 force/torque sensor ${ }^{4}$. As expected, the relation between rotational speed and generated force/torque can be well approximated by a quadratic function. The maximum attainable force and torque are $9.0[\mathrm{~N}]$ and $0.141[\mathrm{Nm}]$ respectively. The response from speed command to actual propeller speed was well representable by a first order linear system with a time constant of 0.047 [sec].

The avionics of the UAV is composed of two main parts: an onboard computer and a microcontroller. The 8-bit Atmega1284p microcontroller, clocked at $20 \mathrm{MHz}$, is able to send the desired motor speeds to the brushless controller by means of an $\mathrm{I}^{2} \mathrm{C}$ bus. It also receives data from a 3D LIS344alh accelerometer $\left(0.0039 g_{0}\left[\mathrm{~m} / s^{2}\right]\right.$ resolution and $\pm 2 g_{0}\left[\mathrm{~m} / \mathrm{s}^{2}\right]$ range) and 3 ADXRS610 gyros $(0.586[\%$ s] resolution and $\pm 300[\% \mathrm{~s}]$ range). The onboard computer is a small Q7 board $^{5}$ with a Z530 Intel Atom processor, 1GB DDR2 $533 \mathrm{MHz}$ RAM, an $8 \mathrm{~GB}$ Flash Disk and a WiFi card, with a total

\footnotetext{
${ }^{3}$ http://www.mikrokopter.de

${ }^{4}$ http://www.ati-ia.com/

${ }^{5}$ http://www.seco.it/en/, http://www.qseven-standard.org/
}
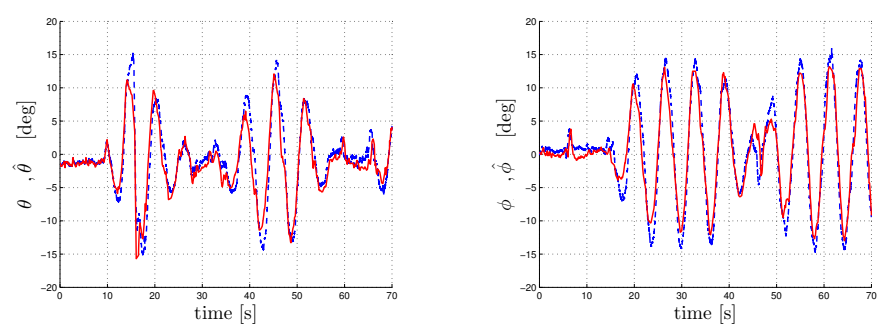

Fig. 4. Roll and pitch angles estimate by the complementary filter (red) and their ground truth values (blue) in a typical experiment.

power consumption of $10[\mathrm{~W}]$. The onboard computer and the microcontroller communicate through a serial (RS232) cable with baud-rate up to $115200[\mathrm{bits} / \mathrm{s}]$. The UAV is also equipped with a low-cost monocular camera, connected to the onboard computer through USB. A set of reflective markers are also attached on it, which is used by an external motion tracking system to retrieve the current position/orientation of the quadrotors.

We used a commercial Omega. $3^{6}$ as haptic master device, with 3 fully-actuated translational degrees of freedom. See Fig. 3. The maximum device force is about $10[\mathrm{~N}]$ and its workspace is cube-shaped with an edge of $0.12[\mathrm{~m}]$. The device is connected to a computer through USB with $2.5 \mathrm{kHz}$ servorate. This computer then communicates to the UAV's onboard compute over an Internet communication.

\section{B. Quadrotor Control and Estimation}

The inner/outer-loop controller explained in Sec. II-B is used for each quadrotor to track its own VP. The faster inner-loop attitude control (9) is run by the microcontroller at a frequency of $500 \mathrm{~Hz}$ while the slower outer-loop position control (7)-(8) is run on the onboard computer at a frequency of about $120 \mathrm{~Hz}$.

The position/orientation data provided by the motion tracking system is directly used by the position controller. However, the update rate of the roll/pitch measurements from the motion tracking system is too slow to be fed back to the (faster) attitude controller. To address this issue, we utilized the standard complementary filters (see, e.g., [42]) to produce a high-rate estimate of the roll and pitch angles by data-fusing the gyroscope and accelerometer readings. The dynamics of the employed complementary filters (valid for small angles and accelerations) are given as follows:

$$
\dot{\hat{\phi}}=\bar{w}_{\phi}+k\left(\bar{a}_{\phi}-\hat{\phi}\right), \quad \dot{\hat{\theta}}=\bar{w}_{\theta}+k\left(\bar{a}_{\theta}-\hat{\theta}\right)
$$

where $\bar{w}$ and $\bar{a}$ are the gyroscope and accelerometer readings influencing the roll and pitch dynamics, and $k$ is a positive gain. Typical performance of this filter is shown in Fig. 4.

\section{Software Setup}

The software for our semi-autonomous teleoperation system consists of several processes interconnected though custom interfaces, as depicted in Fig. 5. A C++ algorithmic library provides the signal processing and control methods needed by

\footnotetext{
${ }^{6} \mathrm{http}: / / \mathrm{www}$. forcedimension.com
} 


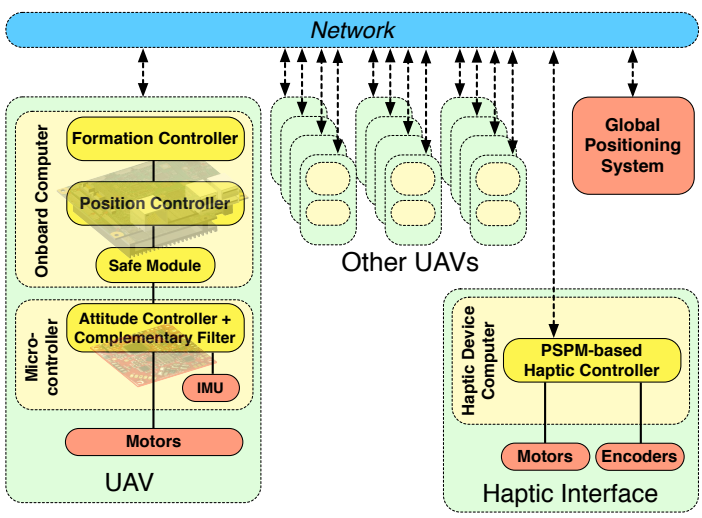

Fig. 5. Software implementation architecture

each process, such as flight control, signal filtering, collective behavior, and force-feedback control.

The microcontroller runs a single process implementing the attitude controller and complementary filter. The onboard computer runs a process implementing the position controller, VP simulation, and another processes for the collective control among the UAVs and communication with the human operator. This onboard computer uses WiFi to communicate via Socket IPC with: 1) the other UAVs' onboard computers; 2) the master haptic device computer; and 3) the external motion tracking system. The haptic device computer runs a local control loop implementing the PSPM algorithm and computing the force cues for the human operator at a frequency of $2.5 \mathrm{kHz}$.

The Safe Module process, a compact and well-tested custom-built program, is also implemented to mediate the communication between the position and attitude controllers with the aim of taking full control of the UAV in the case of detection of some malfunctioning (e.g., erroneous frequencies, excessive jitter, etc.).

\section{Illustrative Experiments}

Using the testbed described so far, we conducted experiments to illustrate the theoretical framework presented in this paper. For this, we set $\mathcal{N}_{t}=\mathcal{N}_{s}$ and $K_{f}=0$ as explained after Th. 1 in Sec. II-D (i.e., in steady-state, haptic feedback solely due to obstacles or visual feedback of collective velocity). In the following, we present the results of two representative ${ }^{7}$ experiments. We also invite readers to watch the attached video where these experiments are shown together with additional materials.

For the first experiment, we design the inter-VP potentials $\varphi_{i j}^{c}$ in (11) to let the four UAVs make a square formation with $2[\mathrm{~m}]$ edge in free space (i.e., no obstacles). A narrow passage with $2.5[\mathrm{~m}]$ clearance is also installed in the middle of the arena. The human user then tele-pushes the team of four UAVs through this passage with haptic feedback several times to show the overall system behavior. See Fig. 6 for some screenshots from a similar experiment.

\footnotetext{
${ }^{7}$ Numerous demonstrations of our teleoperation framework have been performed at the authors' institutions and also at the 2012 International Conference on Intelligent \& Autonomous Systems between Korea and Germany, with the system behaving as postulated by the theory (e.g., Th. 1).
}

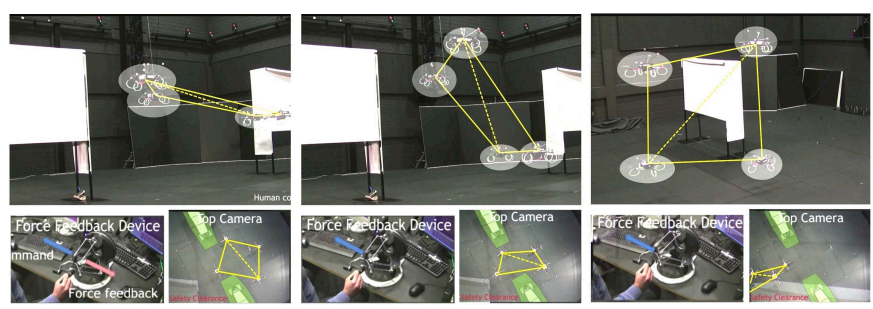

Fig. 6. Screenshot from the first experiment: potentials are designed to render a square formation; human user is tasked to guide the UAVs into a narrow passage.

The first four plots of Fig. 7 respectively shows: 1) the human velocity command $u_{i}^{t}$;2) the average obstacle avoidance action $\left.(1 / 4) \sum_{i=1}^{4} u_{i}^{o} ; 3\right)$ the average velocity of the UAVs $(1 / 4) \sum_{i=1}^{4} \dot{x}_{i}$; and 4) the control torque $\tau$ (i.e., forcefeedback from (3)) provided to the user, with the three lines (red, green and blue) of each plot representing their components in the three orthogonal axes. From there, we can then observe that high force-feedback corresponds to rapid changes in the velocity command (i.e., haptic perception of the velocity mismatch - see Sec. II-D) or to the high values of the obstacle gradient (i.e., haptic obstacle perception: note the opposite signs of $u_{i}^{t}$ and $(1 / 4) \sum_{i=1}^{4} u_{i}^{o}$ as predicted in (22)). Also, note that, in steady-state around $10[\mathrm{sec}]$ with almost-zero obstacle actions, as shown in the item 2-(a) of Th. 1, the average velocity follows the human command with zero control torque (i.e., visual feedback (19) with zero haptic feedback (20)).

The very bottom plot of Fig. 7 contains the evolution of the inter-distances among the UAVs, $\left\|x_{i}-x_{j}\right\|, i, j \in\{1,2,3,4\}$. Given the chosen square formation, in free space, the four distances should be $2[\mathrm{~m}]$ while the remaining two (diagonals) distances $2 \sqrt{2}[\mathrm{~m}]$. These nominal values are plotted with dashed horizontal lines. We can then see there that, due to the presence of obstacles and the teleoperation commands, the actual inter-UAV distances deviate from the nominal ones during the operation, yet, with no collisions/separations among the UAVs. Notice also the correspondence between the phases of large inter-UAV distance errors and high force-feedback in Fig. 7 (i.e., haptic obstacle perception).

Fig. 8 shows the VP-UAV position tracking error $\left\|p_{i}-x_{i}\right\|$, $i=1, . ., 4$, which are fairly small (i.e., less than $5 \%$ of the undeformed inter-distance $2[\mathrm{~m}]$ among the UAVs in Fig. 7). Similar UAV-VP coordination errors have been observed in all the other trials of the experimental campaign. This small VPUAV error then implies that our (practical) trajectory tracking controller in Sec. II-B works properly and our assumption of small $\left\|\dot{x}_{i}-p_{i}\right\|$ in Sec. II-B is indeed valide for the experiment (i.e., VP behaviors and UAV behaviors are equivalent). This, along with Fig. 7, also manifests the stable behavior of our multi-UAV teleoperation system.

In Fig. 9, we also present the trajectories of four UAVs during the first 10 [sec] of the experimental trial. For better presentation while avoiding unnecessary overlaps, here, we report only the results where the fleet of the UAVs is forced by the human user to pass through the narrow opening only once. From Fig. 9, we can then see that the UAVs' formation 

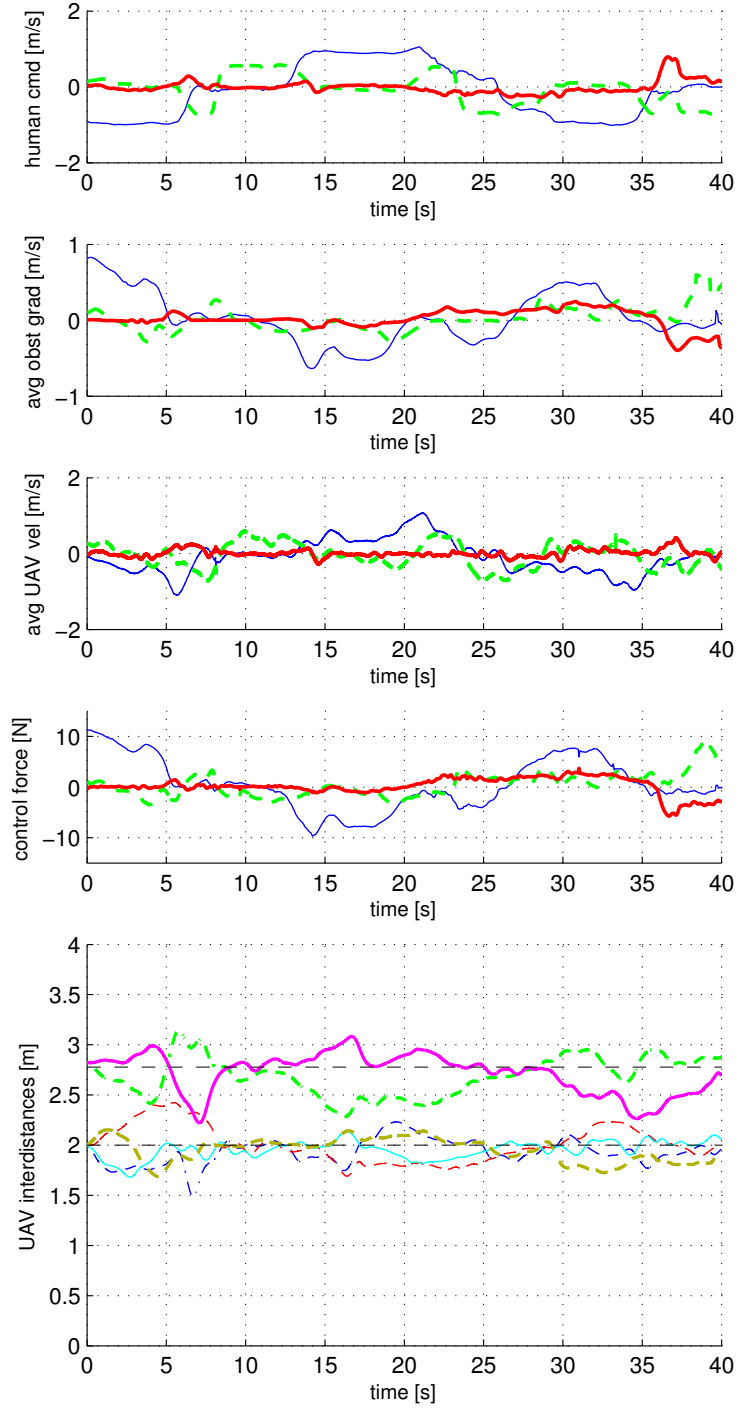

Fig. 7. Human velocity command $u_{i}^{t}$, collective obstacle avoidance gradients $\sum_{i=1}^{4} u_{i}^{o}$, collective UAVs' velocity $\sum_{i=1}^{4} \dot{x}_{i}$, control torque of the master device $\tau$, and inter-distances among the UAVs $\left\|x_{i}-x_{j}\right\|$.

shape deforms during the transition and comes back to the undeformed one after traversing the narrow passage. We can also consider this phase together with the first 10[sec] of Fig. 7, where it is clear how the inter-UAV distances are first deviated from the nominal values and then restored (at approximately $9[\mathrm{sec}]$ ). Finally notice from Fig. 9 (or Fig. 6) that, due to the rotational symmetry of our controller, the square formation shape of the UAVs rotates in $\mathrm{E}(3)$ while interacting with the environment.

To conclude the section, Fig. 10 shows screenshots of the other representative experiment where we designed the VP-VP potential $\varphi_{i j}^{c}$ to generate a tetrahedron formation at rest with a ground obstacle. From the four snapshots in Fig. 10, we can then see that, as the human user tele-drives the UAVs over the obstacle, the whole UAVs' formation rolls over the obstacle, again due to the rotational symmetry of the VP-VP potentials.
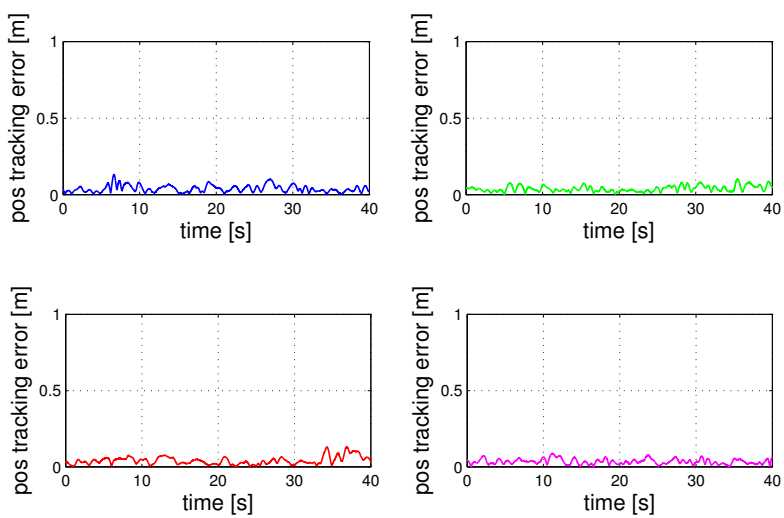

Fig. 8. VP-UAV position tracking errors for each $\mathrm{UAV}\left\|p_{i}-x_{i}\right\|, i=1 \ldots 4$.
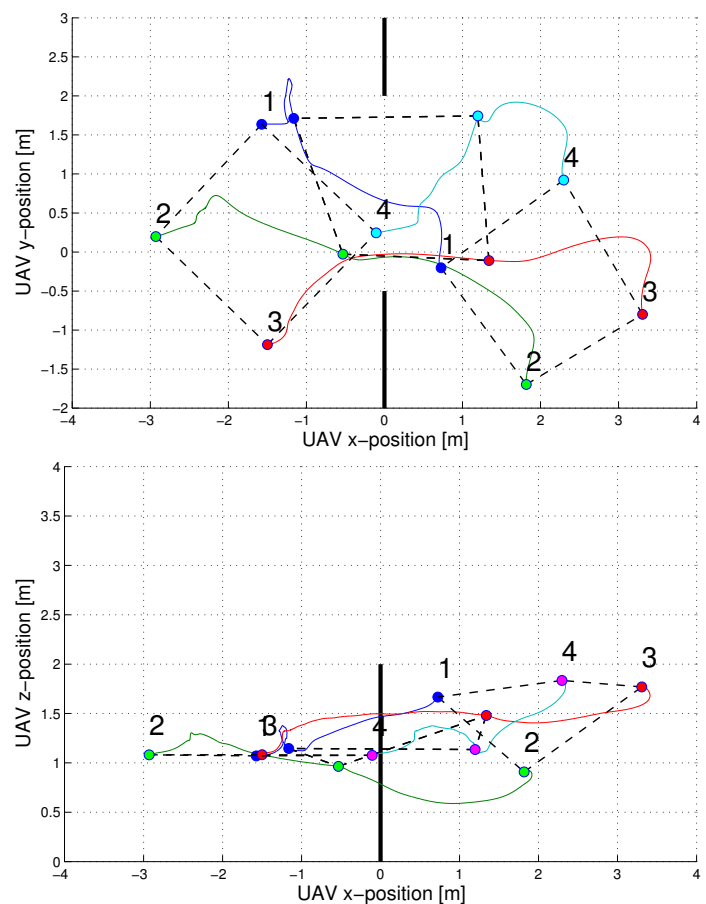

Fig. 9. Trajectories of UAVs projected on the $\mathrm{XY}$ and $\mathrm{XZ}$ planes during the time interval $[0 \mathrm{~s}, 10 \mathrm{~s}]$ : the dashed lines and big dots illustrate the formation and locations of the UAVs at $0 \mathrm{~s}, 5 \mathrm{~s}$, and $10 \mathrm{~s}$, while the black thick lines represent the narrow passage gap.

\section{Summary And Future Research}

We proposed a novel haptic teleoperation control framework for multiple UAVs, consisting of three layers: 1) UAV control layer to drive each UAV to follow its own VP; 2) VP control layer to render $N$ VPs as a deformable flying object with inter-VP/VP-obstacle collision avoidance and inter-VP connectivity preservation; and 3) PSPM-based teleoperation layer to allow a human user to tele-control the bulk motion of $N$ VPs with some useful haptic feedback over the Internet. Master-passivity/slave-stability and some asymptotic performance measures are proved. Experiment results are also presented.

Some possible future research directions include: 1) reduction of the number of UAVs directly communicating with the master while retaining the same level of performance (e.g., the same level of controllability [43]); 2) elimination of VPs 


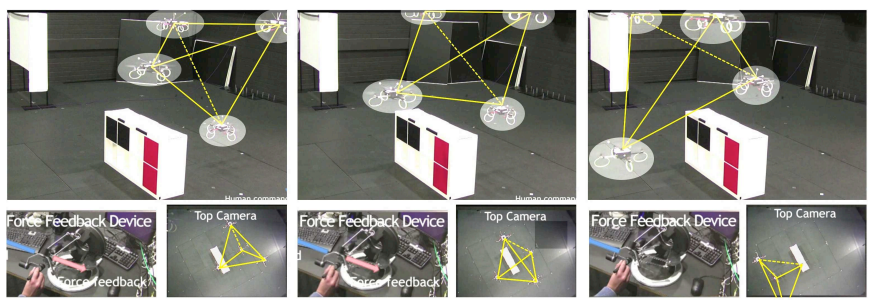

Fig. 10. Screenshot from the second experiment: potentials are designed to make a tetrahedron formation; human user is tasked to guide the UAVs over a ground obstacle.

altogether (see [44] for preliminary results in this direction); 3) application to a real task with the haptic feedback (15) perceptually-optimized for that task (by using the method of [27], [41]); and 4) experimental comparison with other semiautonomous teleoperation control techniques.

\section{REFERENCES}

[1] K. P. Valavanis, editor. Advances in Unmanned Aerial Vehicles: State of the Art and the Road to Autonomy. Springer, 2007. Intelligent Systems, Control and Automation: Science and Engineering, Vol. 33.

[2] G. Vachtsevanos and K. Valavanis, editors. IEEE Robotics and Automation Magazine: Special Issue on Unmanned Aerial Vehicles, volume 13. September 2006.

[3] T. J. Koo and S. Sastry. Output tracking control design of a helicopter model based on approximate linearization. In Proc. IEEE Conference on Decision \& Control, pages 3635-3640, 1998.

[4] R. Mahony and T. Hamel. Robust trajectory tracking for a scale model autonomous helicopter. International Journal of Robust and Nonlinear Control, 14:1035-1059, 2004.

[5] A. P. Aguiar and J. P. Hespanha. Trajectory-tracking and path-following of underactuated autonomous vehicles with parametric modeling uncertainty. IEEE Transactions on Automatic Control, 52(8):1362-1379, 2007.

[6] M-D. Hua, T. Hamel, P. Morin, and C. Samson. A control approach for thrust-propelled underactuated vehicles and its application to vtol drones. IEEE Transactions on Automatic Control, 54(8):1837-1853, 2009.

[7] D. J. Lee, C. Ha, and Z. Zuo. Backstepping control of quadrotor-type uavs: trajectory tracking and teleoperation over the internet. In Proc. Int'l Conf. on Autonomous Systems, pages 217-225, June 2013.

[8] J. M. Pflimlin, P. Soueres, and T. Hamel. Position control of a ducted fan vtol uav in crosswind. International Journal of Control, 80(5):666-683, 2007.

[9] M. Oishi and C. J. Tomlin. Switched nonlinear control of a vstol aircraft. In Proc. IEEE Conf. on Decision \& Control, pages 2685-2690, 1999.

[10] D. J. Lee and K. Huang. Passive-set-position-modulation framework for interactive robotic systems. IEEE Transactions on Robotics, 26(2):354 $369,2010$.

[11] T. M. Lam, M. Mulder, and M. M. van Paassen. Haptic feedback in uninhabited aerial vehicle teleoperation with time delay. AIAA Journal of Guidance, Control \& Dynamics, 31(6):1728-1739, 2008.

[12] S. Stramigioli, R. Mahony, and P. Corke. A novel approach to haptic tele-operation of aerial robot vehicles. In Proc. IEEE Int'l Conf. on Robotics \& Automation, pages 5302-5308, 2010.

[13] C. Masone, A. Franchi, H. H. Bülthoff, and P. Robuffo Giordano. Interactive planning of persistent trajectories for human-assisted navigation of mobile robots. In 2012 IEEE/RSJ Int. Conf. on Intelligent Robots and Systems, pages 2641-2648, 2012.

[14] D. J. Lee and M. W. Spong. Bilateral teleoperation of multiple cooperative robots over delayed communication networks: theory. In Proc. IEEE Int'l Conf. on Robotics \& Automation, pages 362-367, 2005.

[15] E. J. Rodriguez-Seda, J. J. Troy, C. A. Erignac, P. Murray, D. M. Stipanovic, and M. W. Spong. Bilateral teleoperation of multiple mobile agents: coordinated motion and collision avoidance. IEEE Transactions on Control Systems Technology, 18(4):984-992, 2010.

[16] D. J. Lee and P. Y. Li. Passive decomposition of mechanical systems with coordination requirement. IEEE Transactions on Automatic Control, 58(1):230-235, 2013.

[17] D. J. Lee and P. Y. Li. Passive decomposition approach to formation and maneuver control of multiple rigid bodies. Journal of Dynamic Systems, Measurement \& Control, 129:662-677, September 2007.
[18] D. J. Lee and D. Xu. Feedback $r$-passivity of lagrangian systems for mobile robot teleoperation. In Proc. IEEE Int'l Conference on Robotics \& Automation, pages 2118-2123, 2011.

[19] D. J. Lee. Passive decomposition and control of nonholonomic mechanical systems. IEEE Transactions on Robotics, 26(6):978-992, 2010.

[20] D. J. Lee. Semi-autonomous teleoperation of multiple wheeled mobile robots over the internet. In Proc. ASME Dynamic Systems \& Control Conference, pages 147-154, 2008.

[21] D. J. Lee, A. Franchi, P. Robuffo Giordano, H-I. Son, and H. H. Bülthoff. Haptic teleoperation of multiple unmanned aerial vehicles over the internet. In Proc. IEEE Int'l Conference on Robotics \& Automation, pages 1341-1347, 2011.

[22] A. Franchi, P. Robuffo Giordano, C. Secchi, H. I. Son, and H. H. Bülthoff. A passivity-based decentralized approach for the bilateral teleoperation of a group of uavs with switching topology. In Proc. IEEE Int'l Conf. on Robotics \& Automation, pages 898-905, 2011.

[23] A. Franchi, C. Secchi, H. I. Son, H. H. Bülthoff, and P. Robuffo Giordano. Bilateral teleoperation of groups of mobile robots with timevarying topology. IEEE Transactions on Robotics, 28(5):1019-1033, 2012.

[24] C. Secchi, A. Franchi, H. H. Bülthoff, and P. Robuffo Giordano. Bilateral teleoperation of a group of UAVs with communication delays and switching topology. In 2012 IEEE Int. Conf. on Robotics and Automation, pages 4307-4314, St. Paul, MN, May 2012.

[25] A. Franchi, C. Masone, V. Grabe, M. Ryll, H. H. Bülthoff, and P. Robuffo Giordano. Modeling and control of UAV bearing-formations with bilateral high-level steering. International Journal of Robotics Research, 31(12):1504-1525, 2012.

[26] P. Robuffo Giordano, A. Franchi, C. Secchi, and H. H. Bülthoff. A Passivity-based decentralized strategy for generalized connectivity maintenance. International Journal of Robotics Research, 32(3):299323, 2013.

[27] H. I. Son, A. Franchi, L. L. Chuang, J. Kim, H. H. Bülthoff, and P. Robuffo Giordano. Human-centered design and evaluation of haptic cueing for teleoperation of multiple mobile robots. IEEE Trans. on Systems, Man, \& Cybernetics. Part B: Cybernetics, 43(2): 597-609, 2013.

[28] K. Huang and D. J. Lee. Implementation and experiments of passive setposition modulation framework for interactive robotic systems. In Proc. IEEE/RSJ Int'l Conf. on Intelligent Robots \& Systems, pages 5615-5620, 2009.

[29] A. Franchi, C. Secchi, M. Ryll, H. H. Bülthoff, and P. Robuffo Giordano. Shared control: Balancing autonomy and human assistance with a group of quadrotor uavs. IEEE Robotics \& Automation Magazine, 19(3), 2012.

[30] K. Tanaka, H. Ohtake, M. Tanaka, and H. O. Wang. Wireless visionbased stabilization of indoor microhelicopter. IEEE/ASME Trans. on Mechatronics, 17(3): 519-524, 2012.

[31] M. W. Spong, S. Hutchinson, and M. Vidyasaga. Robot modeling and control. John Wiley \& Sons, Hoboken, NJ, 2006.

[32] J. Aspnes, T. Eren, D. K. Goldenberg, A. S. Morse, W. Whiteley, B. D. O. Anderson, and P. N. Belhumeur. A theory of network localization. IEEE Transactions on Mobile Computing, 5(12):1663 $1678,2006$.

[33] D. Zelazo, A. Franchi, F. Allgöwer, H. H. Bülthoff, and P. Robuffo Giordano. Rigidity maintenance control for multi-robot systems. In 2012 Robotics: Science and Systems, Sydney, Australia, Jul. 2012.

[34] D. V. Dimarogonas and K. J. Kyriakopoulos. Connectedness preserving distributed swam aggregation for multiple kinematic robots. IEEE Transactions on Robotics, 24(5):1213-1223, 2008.

[35] P. Ogren, E. Fiorelli, and N. E. Leonard. Cooperative control of mobile sensor networks: Adaptive gradient climbing in a distributed environment. IEEE Transactions on Automatic Control, 49(8):1292$1302,2004$.

[36] Y. Cao and W. Ren. Distributed coordinated tracking via a variable structure approach - part ii: swarm tracking. In Proc. of the American Control Conference, pages 4750-4755, 2010.

[37] A. Sarlette, R. Sepulchre, and N. E. Leonard. Cooperative attitude synchronization in satellite swarms: a consensus approach. In Proc. 17th IFAC Symp. on Automatic Control in Aerospace, 2007.

[38] O. M. Palafox and M. W. Spong. Bilateral teleoperation of a formation of nonholonomic mobile robots under constant time delay. In Proc. IEEE/RSJ Int'l Conf. on Intelligent Robots \& Systems, pages 2821-2826, 2009.

[39] S. F. F. Gibson and B. Mirtich. A survey of deformable modeling in computer graphics. In MERL Technical Report, Cambridge, MA, 1997. Mitsubishi Electric Information Technology Center America. 
[40] C. W. Reynolds. Flocks, herds, and schools: a distributed behavioral model. Computer Graphics, 21(4):25-34, 1987.

[41] H. I. Son, L. L. Chuang, J. Kim, and H. H. Bülthoff. Haptic feedback can improve human perceptual awareness in multi-robots teleoperation. In Proc. Int'l Conf. on Control, Automation \& Systems, pages 1323-1328, 2011.

[42] R. Mahony, T. Hamel, and J.-M. Pflimlin. Complementary filter design on the special orthogonal group SO(3). In Proc. IEEE Conf. on Decision \& Control, pages 1477-1484, 2005.

[43] M. Egerstedt, S. Martini, M. Cao, K. Camlibel, and A. Bicchi. Interacting with networks. IEEE Control Systems Magazine, 32(4):66-73, 2012.

[44] D. J. Lee. Distributed backstepping control of multiple thrust-propelled vehicles on balanced graph. Automatica, 48(11):2971-2977, 2012.

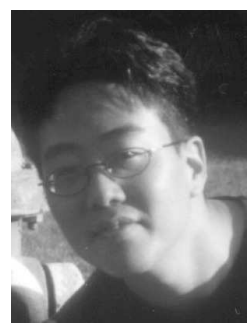

Dongjun Lee (S'02-M'04) received the Ph.D. degree in mechanical engineering from the University of Minnesota at Twin Cities in 2004. Since 2011, he has been an Assistant Professor with the School of Mechanical \& Aerospace Engineering at Seoul National University, Korea. He was an Assistant Professor with the Department of Mechanical, Aerospace and Biomedical Engineering at the University of Tennessee from 2006 to 2011, and a Postdoctoral Researcher with the Coordinated Science Lab at the University of Illinois at Urbana-Champaign, from 2004 to 2006. His main research interests are dynamics and control of robotic and mechatronic systems with emphasis on teleoperation/haptics, multirobot systems, aerial robots, and geometric mechanics control theory. Dr. Lee received the US NSF CAREER Award in 2009 and is an Associate Editor of the IEEE Transactions on Robotics.

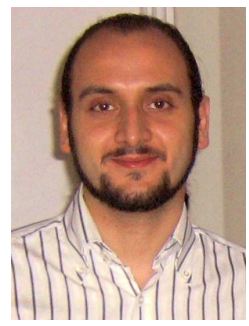

Antonio Franchi (S'07-M'11) received the Laurea degree (summa cum laude) in electronic engineering and the $\mathrm{Ph} . \mathrm{D}$. degree in control and system theory from the Sapienza University of Rome, Italy, in 2005 and 2009, respectively. He was a visiting student with the University of California at Santa Barbara, in 2009. In 2010, he joined the Max Planck Institute for Biological Cybernetics, Tübingen, Germany, where he is currently a Senior Research Scientist, Head of the Autonomous Robotics and Human Machine Systems group. He is Associate Editor of the IEEE Robotics and Automation Magazine. His main research interests include autonomous systems and robotics, with a special regard to control, planning, estimation, human-machine interaction, haptics, and hardware/software architectures. He published over 50 papers in these areas.

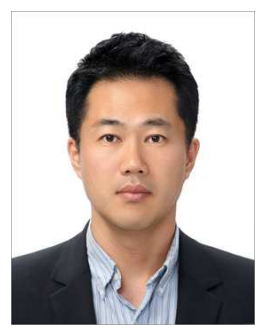

Hyoung Il Son (M11) received the B.S. and M.S. degrees from the Department of Mechanical Engineering, Pusan National University, Busan, Korea, in 1998 and 2000, respectively, and the Ph.D. degree from the Department of Mechanical Engineering, KAIST (Korea Advanced Institute of Science and Technology), Daejeon, Korea in 2010. He is currently a Principal Researcher at the Institute of Industrial Technology, Samsung Heavy Industries, Daejeon, Korea. Before joining Samsung Heavy Industries, he was a Research Scientist with the Max Planck Institute for Biological Cybernetics, Tubingen, Germany. He was a Senior Researcher at LG Electronics (2003-2005) and Samsung Electronics (2005-2009), and a Research Associate at the Institute of Industrial Science, the University of Tokyo, Tokyo, Japan (2010). His research interests include haptics, teleoperation, underwater robotics, psychophysics, and supervisory control of discrete event/hybrid systems.

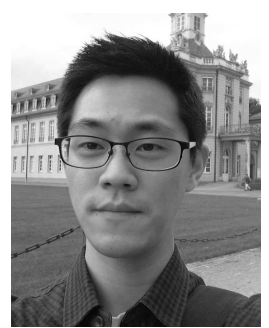

ChangSu Ha (S'13) received the B.S. degree in mechanical engineering from Sungkyunkwan University, Suwon, Korea, in 2002. He is currently working toward the M.S. degree in mechanical engineering from Seoul National University, Seoul, Korea. His research interests include Internet teleoperation and control of flying robots.

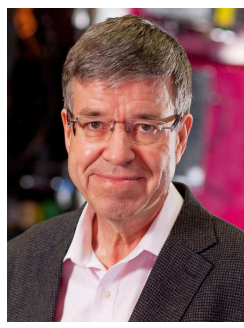

Heinrich H. Bülthoff (M'96) completed his Ph.D. thesis in Biology at the Eberhard Karls University in Tübingen, Germany in 1980. From 1980 to 1988 he worked as a research scientist at the Max Planck Institute for Biological Cybernetics and the Massachusetts Institute of Technology (MIT). He was Assistant, Associate and Full Professor of Cognitive Science at Brown University in Providence from 1988-1993 before becoming director of the Department for Human Perception, Cognition and Action at the Max Planck Institute for Biological Cybernetics and a scientific member of the Max Planck Society in 1993. Heinrich Bülthoff is Honorary Professor at the Eberhard Karls University (Tübingen, Germany) since 1996 as well as Adjunct Professor at the Korea University (Seoul, Korea). His research interests include object recognition and categorization, perception and action in virtual environments, human-robot interaction and perception.

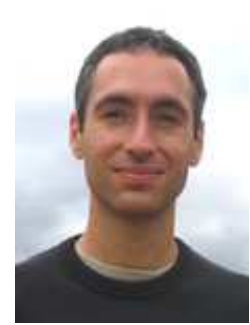

Paolo Robuffo Giordano (M'08) received the M.Sc. degree in Computer Science Engineering in 2001, and the Ph.D. degree in Systems Engineering in 2008, both from the University of Rome "La Sapienza". Between 2007 and 2008 he spent one year and half as a PostDoc at the Institute of Robotics and Mechatronics of the German Aerospace Center, and from 2008 to 2012 he was Senior Research Scientist head of the Human-Robot Interaction group at the Max Planck Institute for Biological Cybernetics. His research interests span nonlinear control, robotics, haptics and VR applications. 